\title{
Ionotropic Glutamate Receptors in the Ventral Tegmental Area Regulate Cocaine-Seeking Behavior in Rats
}

\author{
WenLin Sun', Chana K Akins ${ }^{2}$, Anne E Mattingly' and George V Rebec*, \\ 'Program in Neural Science, Department of Psychology, Indiana University, Bloomington, IN, USA; ${ }^{2}$ Department of Psychology, University of \\ Kentucky, Lexington, KY, USA
}

\begin{abstract}
Drug addiction is characterized by compulsive drug-seeking and drug-taking behavior and by a high rate of relapse even after long periods of abstinence. Although the mesocorticolimbic dopamine (DA) pathway is thought to play a critical role in drug craving and relapse, recent evidence also implicates glutamate, an amino acid known to activate DA neurons in the ventral tegmental area ( $V T A$ ) via ionotropic receptors. To assess whether increased glutamate transmission in the VTA is involved in cocaine-primed drug-seeking behavior, we tested rats in a between-session reinstatement model. They were trained to press a lever for cocaine infusions $(0.25 \mathrm{mg} /$ infusion) accompanied by compound stimuli (light and tone) under a modified fixed-ratio 5 reinforcement schedule. Cocaine-primed reinstatement was conducted after lever pressing was extinguished in the absence of the conditioned stimuli. Blockade of ionotropic glutamate receptors in the VTA by local application of kynurenate $(0.0,1.0,3.2$, and $5.6 \mu \mathrm{g} / \mathrm{side})$ dose-dependently decreased cocaineprimed reinstatement, whereas sucrose-primed reinstatement of sucrose-seeking behavior was unaffected. In addition, the minimum effective dose for decreasing cocaine-primed reinstatement was ineffective in the substantia nigra. Together, these data indicate that glutamatergic activation of the VTA is critical for cocaine-primed reinstatement. Because such activation can increase impulse flow in DA neurons and thus DA release in mesocorticolimbic targets, this glutamate-DA interaction in the VTA may underlie cocaine-primed relapse to cocaine-seeking behavior.

Neuropsychopharmacology (2005) 30, 2073-208I. doi:I0.I038/sj.npp. I 300744; published onlilne 20 April 2005
\end{abstract}

Keywords: cocaine; self-administration; reinstatement; kynurenate; ventral tegmental area; mesocorticolimbic dopamine; glutamate

\section{INTRODUCTION}

Drug addiction is characterized by repeated occurrences of drug craving and relapse even after long periods of abstinence (Leshner and Koob, 1999). It is widely believed that these long-lasting behavioral changes are caused by drug-induced adaptations in brain regions involved in reward, motivation, and learning and memory (Hyman and Malenka, 2001; McClung and Nestler, 2003; Nestler, 2004). Understanding the mechanisms by which these brain regions contribute to drug craving and relapse is critical for developing an effective therapy for drug addiction.

The mesocorticolimbic circuit, which arises from dopamine (DA) neurons in the ventral tegmental area (VTA) and includes the forebrain regions that receive DA input such as the nucleus accumbens (NAcc) and prefrontal cortex (PFC), appears to be the critical substrate of drug craving and relapse. Imaging studies of cocaine addicts show that both

\footnotetext{
*Correspondence: Dr GV Rebec, Program in Neural Science, Department of Psychology, Indiana University, II0I E IOth Street, Bloomington, IN 47405, USA, Tel: + I 812855 4832, Fax: + I 812 855 4520, E-mail: rebec@indiana.edu

Received 23 September 2004; revised and accepted 28 February 2005 Online publication: 4 March 2005 at http://www.acnp.org/citations/ NPP030405040445/default.pdf
}

cocaine and cocaine-paired cues increase metabolic activity in this circuit (Maas et al, 1998; Kilts et al, 2001; Grant et al, 1996; Breiter et al, 1997; Childress et al, 1999). Preclinical studies based on reinstatement models also point to the important role of this circuit in cocaine-seeking behavior (McFarland and Kalivas, 2001; Capriles et al, 2003; McLaughlin and See, 2003). In fact, preclinical evidence has identified specific receptor mechanisms by which various components of the mesocorticolimbic circuit control behavioral responses to cocaine. For example, DA receptors in the PFC and ionotropic glutamate (iGLU) receptors in the NAcc seem critical for cocaine-primed drug-seeking behavior (Cornish and Kalivas, 2000; McFarland and Kalivas, 2001; Park et al, 2002; Sun and Rebec, 2005).

Given the important role of DA in cocaine-primed reinstatement, it is expected that the VTA, the source of DA in the mesocorticolimbic circuit, also plays a role in this process. Indeed, infusion of morphine into the VTA induces cocaine-seeking behavior in rats after extinction of responding maintained by cocaine (Stewart, 1984). The authors suggest that increased DA neuronal activity resulting from morphine-mediated disinhibition of DA neurons (Johnson and North, 1992) may be responsible for this effect. In addition, infusion of $N$-methyl-D-aspartate 
(NMDA) into the VTA reinstates cocaine-seeking behavior presumably by activating NMDA receptors, a subtype of iGLU receptors (Vorel et al, 2001) on DA neurons. Together, these data indicate that activation of DA neurons in the VTA may trigger cocaine-seeking behavior by increasing DA neurotransmission in forebrain structures. It is unclear, however, whether this activation is necessary for cocaine-primed drug-seeking behavior because with a basal level of DA impulse flow cocaine itself can increase extracellular DA levels in the forebrain. If cocaine-primed reinstatement depends on activation of the VTA, it is likely that the information related to cocaine priming is relayed to the VTA from other brain regions.

Several regions including PFC, amygdala, the midbrain tegmental nuclei, and the bed nucleus of the stria terminalis project directly to the VTA (Sesack et al, 2003; Georges and Aston-Jones, 2002). Most of these inputs are glutamatergic and excite DA neurons (Takahata and Moghaddam, 1998; Almodovar-Fabregas et al, 2002; Georges and Aston-Jones, 2002). In addition, iGLU receptors mediate the ability of cocaine to elicit long-term potentiation, a putative mechanism for learning, in DA neurons (Ungless et al, 2001). Together, these data indicate an involvement of VTA glutamate in cocaine effects. It is likely, therefore, that cocaine priming may reinstate drug-seeking behavior by increasing glutamate inputs into the VTA.

To test this hypothesis, we studied the effects of blockade of iGLU receptors in the VTA by infusion of kynurenate (KYN), a nonselective iGLU receptor antagonist (Stone, 1993), on cocaine-seeking behavior in rats using a betweensession model of reinstatement. KYN was selected because it inhibits glutamate-mediated activation of VTA DA neurons without significantly altering basal activity (Georges and Aston-Jones, 2002). We also evaluated the behavioral specificity of iGLU receptor blockade by studying KYN effects on cocaine self-administration (SA) as well as on sucrose-maintained and sucrose-reinstated operant responding. Separate groups of rats, moreover, received infusions of KYN into the substantia nigra (SN), which is adjacent to the VTA and houses DA neurons that project primarily to structures in the basal ganglia. The data from this group will help us to evaluate whether diffusion of KYN from the VTA into the SN contributes to our results.

\section{MATERIALS AND METHODS}

\section{Subjects}

Male Sprague-Dawley rats (350-400g), bred from rats supplied by Harlan Industries (Indianapolis, IN), were obtained from the departmental breeding colony. They were housed individually in plastic home cages in a temperatureand humidity-controlled colony room on a 12-h light-dark cycle (lights on at 0730). At 1 week before operant training, rats were placed on a restricted diet to reach $\sim 85 \%$ freefeeding weight. After training, free access to food was available for 1 week before and after surgery. Food restriction was then reinstated to maintain $85-90 \%$ of free-feeding weight throughout the experiments. Water was always freely available. The experiments were conducted during the light cycle (between 0900 and 1800). All procedures followed the National Institute of Health
Guidelines for the Care and Use of Laboratory Animals and were approved by the Indiana University Animal Care and Use Committee.

\section{Operant Training}

Rats were first trained to press a lever for sucrose pellets contingent upon a fixed-ratio 1 (FR 1) reinforcement schedule in a standard operant chamber described elsewhere (Sun and Rebec, 2003). Each lever press resulted in delivery of one $45 \mathrm{mg}$ sucrose pellet (Research Diet, New Brunswick, NJ) followed by a 10-s timeout signaled by illumination of the house light. After lever-pressing behavior was established, the reinforcement schedule gradually increased to FR 5 and training continued until rats obtained 60 sucrose pellets within $30 \mathrm{~min}$.

\section{Surgery}

Rats were anesthetized with ketamine ( $80 \mathrm{mg} / \mathrm{kg}$, i.p.) and xylazine $(10 \mathrm{mg} / \mathrm{kg}$, i.p.). Details of catheterization of the right jugular vein are described elsewhere (Caine et al, 1999). Briefly, a catheter constructed from PE10 and PE50 tubing (Fisher Scientific, Pittsburgh, PA) was inserted into the right jugular vein. The PE50 end was inserted over the metal tubing of a guide cannula (22 ga, Plastics One, Roanoke, VA), which was bent at a right angle. The guide cannula was threaded under the skin to exit at the top of the skull. After catheterization, rats were fixed in a stereotaxic apparatus. Two guide cannulas (26 ga, Plastics One, Roanoke, VA) were bilaterally implanted $2 \mathrm{~mm}$ above the VTA and SN. For the VTA, the guide cannulas were angled $10^{\circ}$ away from the midline (VTA: AP: $-5.6 \mathrm{~mm}$, ML: $\pm 1.8 \mathrm{~mm}$, and $\mathrm{DV}:-8.3 \mathrm{~mm}$; SN: AP: $-5.6 \mathrm{~mm}$, ML: $\pm 2.0 \mathrm{~mm}$, and DV: $-8.0 \mathrm{~mm}$, relative to bregma, midline, and skull surface, respectively). Four stainless steel screws were implanted in the skull for support. The cannulas and screws were held in place with dental cement. An obturator (Plastics One, Roanoke, VA) was inserted into each guide cannula to prevent blockage. For the sucrose SA group, the procedure for surgery was the same except that rats were not catheterized. After surgery, all rats were allowed to recover for 1 week during which $0.1 \mathrm{ml}$ (i.v., $10 \mathrm{mg} / \mathrm{ml}$ ) of gentamicin (Biowhitaker, Walkersville, MD) was given daily, and the catheter was flushed twice a day with heparinized physiological saline $(30 \mathrm{U} / \mathrm{ml}$ heparin). During the period of cocaine SA, catheter patency was evaluated by injecting $0.1 \mathrm{ml}$ Brevital (1\%) as necessary. Loss of muscle tone within $5 \mathrm{~s}$ after injection indicates a patent catheter.

\section{Cocaine and Sucrose SA Training}

For cocaine SA, two groups of rats ( $n=8$ for VTA; $n=6$ for $\mathrm{SN}$ ) were trained to press the lever for cocaine in a daily $2-\mathrm{h}$ session contingent upon a modified FR 5 schedule: the first response was reinforced by an infusion of $0.25 \mathrm{mg}$ cocaine in a volume of $0.05 \mathrm{ml}$ accompanied by conditioned stimuli (CS), which consisted of two flashing lights and a tone for $4 \mathrm{~s}$. Delivery of the CS and cocaine was followed by a 16-s timeout period signaled by illumination of the house light. During cocaine infusions and timeouts, responding was recorded but had no programmed consequences. After the 
first response and cocaine infusion, subsequent infusions and presentations of the CS were contingent upon an FR 5 schedule. The session ended when $2 \mathrm{~h}$ passed or 30 infusions of cocaine were delivered, whichever occurred first. After 10-14 days, rats reached training criterion (the number of cocaine infusions varied by $<20 \%$ in three consecutive training sessions). For sucrose SA $(n=8)$, rats were trained under an FR 5 reinforcement schedule in daily 30-min sessions. Every fifth response was reinforced by one sucrose pellet followed by a 10 -s timeout signaled by illumination of the house light. Rats were trained for 5 days per week and the training continued until the response rate varied by $<20 \%$ in three consecutive training sessions. Rats usually reached the criterion within 14 days.

\section{Extinction}

Extinction training was conducted in daily 30 - or 60 -min sessions for the sucrose and cocaine SA groups, respectively. During each extinction session, responding was recorded but had no programmed consequences. Extinction training continued until responding fell below $20 \%$ of the level during cocaine SA or below $5 \%$ of the level during sucrose $\mathrm{SA}$, respectively. These levels were reached in 6-7 days.

\section{Reinstatement}

For cocaine-primed reinstatement, rats were given i.p. injection of $10 \mathrm{mg} / \mathrm{kg}$ cocaine $3 \mathrm{~min}$ before the session started. Lever pressing was reinforced by the CS contingent upon an FR 5 schedule. The session lasted $1 \mathrm{~h}$. An i.p. rather than i.v. injection is preferred for reinstatement testing to eliminate the need to maintain catheter patency for the entire experiment. Our i.p. dose, moreover, is known to reinstate robust cocaine-seeking behavior in rats trained under the same conditions (Sun and Rebec, 2003). For sucrose-induced reinstatement, the session started with noncontingent delivery of a train of three sucrose pellets spaced $10 \mathrm{~s}$ apart, during which the house light was illuminated. Thereafter, the same train was delivered every $3 \mathrm{~min}$. Responding had no programmed consequences during the session, which lasted $30 \mathrm{~min}$. This group was used to evaluate whether KYN infusions nonspecifically affect the motor aspect of reinstated operant responding. Thus, we did not reinforce lever pressing with the CS as in cocaine-primed reinstatement. Informal observation of each animal's behavior was made during the SA, extinction, and reinstatement sessions.

\section{Infusion and Testing Procedures}

The effects of bilateral infusions of $\operatorname{KYN}(0,1,3.2$, and $5.6 \mu \mathrm{g} / \mathrm{side}$ ) on cocaine SA were tested in the VTA and SN groups. Doses were based on pilot studies as well as the literature (Vorel et al, 2001). To minimize diffusion of KYN from the VTA to the SN or vice versa, we used a relatively small injection volume and chose VTA injection sites at least $0.5 \mathrm{~mm}$ away from the SN. Before the SA test sessions, KYN was infused into the VTA or SN in a volume of $0.5 \mu \mathrm{l}$ through 33 ga injection cannulas (Plastics One, Roanoke, VA), which extended $2 \mathrm{~mm}$ below the guide cannula. Infusions occurred over a 1-min period and the injection cannula stayed in place for another minute to ensure drug diffusion. After the infusions, rats were immediately placed in the chambers and the session started 3 min later. The reinforcement schedule was exactly the same as that in the cocaine SA training sessions. Test sessions were conducted on Tuesdays and Fridays. SA training continued between the tests to ensure that responding after tests returned to pretest level. After these experiments, the same groups of rats that went through extinction training were tested with KYN on cocaine-primed reinstatement. Before the reinstatement test sessions, KYN was infused into the VTA or SN followed by i.p. cocaine $(10 \mathrm{mg} / \mathrm{kg})$. Rats were then placed in the chambers and the session started $3 \mathrm{~min}$ later. Reinstatement test sessions were conducted on Tuesdays and Fridays. Extinction training continued between reinstatement tests to ensure that responding after tests returned to pretest extinction levels. KYN doses and saline were administered in a counterbalanced order to ensure that the four different doses were simultaneously tested in each test session to cancel out any order effect. The effects of KYN on sucrose SA and sucrose-primed reinstatement were tested with the same procedures except that only three doses $(0,1$, and $3.2 \mu \mathrm{g} /$ side) were used to avoid possible nonspecific locomotor effects of $5.6 \mu \mathrm{g} /$ side (informal observations).

\section{Histology}

After the experiments, rats were deeply anesthetized with chloropent $(3.3 \mathrm{ml} / \mathrm{kg})$ and transcardially perfused with physiological saline followed by formalin (5\%). The brains were removed, soaked in formalin for at least $24 \mathrm{~h}$, sliced at $60 \mu \mathrm{m}$ thickness, mounted on gelatin-coated slides, and stained with cresyl violet. The positions of the infusion cannula were inspected under a light microscope.

\section{Drugs}

KYN was purchased from Sigma (St Louis, MO) and dissolved in physiological saline to prepare different concentrations $(0-11.2 \mathrm{mg} / \mathrm{ml})$. The $\mathrm{pH}$ of the solutions was adjusted to $7.4 \pm 0.2$ with sodium hydroxide. Cocaine hydrochloride was obtained from the National Institute on Drug Abuse (Bethesda, MD) and was dissolved in physiological saline to prepare a solution with a concentration of $5 \mathrm{mg} / \mathrm{ml}$ (salt).

\section{Statistics}

Responding (lever presses) was recorded during the SA, extinction, and reinstatement sessions. Response rates were calculated as responses per hour for the cocaine SA groups and as responses per minute or $30 \mathrm{~min}$ for the sucrose group. Control data were obtained from the training sessions on Thursdays before the tests. A repeated oneway ANOVA was used for analyzing dose-dependent effects and the effects of different doses were compared with Bonferroni's post-tests. A repeated two-way ANOVA was used for analyzing the time-course effects of different doses on cocaine SA. The time $\times$ dose interaction was compared with Bonferroni's post-tests. The significance level was set at 0.05 . 


\section{RESULTS}

\section{Histology}

The positions of all microinjection sites are shown schematically in Figure 1. All placements were within the VTA or SN.

\section{Experiment 1. Effects of Bilateral Intra-VTA KYN on Cocaine SA}

The time-course effects of different doses of KYN in the VTA on cocaine SA were studied in rats that met the training criteria $(n=8)$. As shown in the upper panel of Figure 2, the 2-h session was divided into six 20 -min bins. A repeated, two-way ANOVA revealed a significant timedose interaction $\left(\mathrm{F}_{15,126}=2.39, P=0.0045\right)$. Bonferroni's tests indicated that both 3.2 and $5.6 \mu \mathrm{g} /$ side significantly decreased responding in the first two, 20-min bins compared with saline pretreatment $(P<0.01)$. KYN had no significant effects on responding during timeout periods (Table 1 , repeated one-way ANOVA, $\mathrm{F}_{3,21}=1.72, P=0.19$ ).

\section{Experiment 2. Effects of Bilateral Intra-SN KYN on Cocaine SA}

The time-course effects of different doses of KYN in the SN were studied in another group of rats $(n=6)$. Due to missing values in the last two bins of the session for one rat, statistical analysis was conducted on the remaining

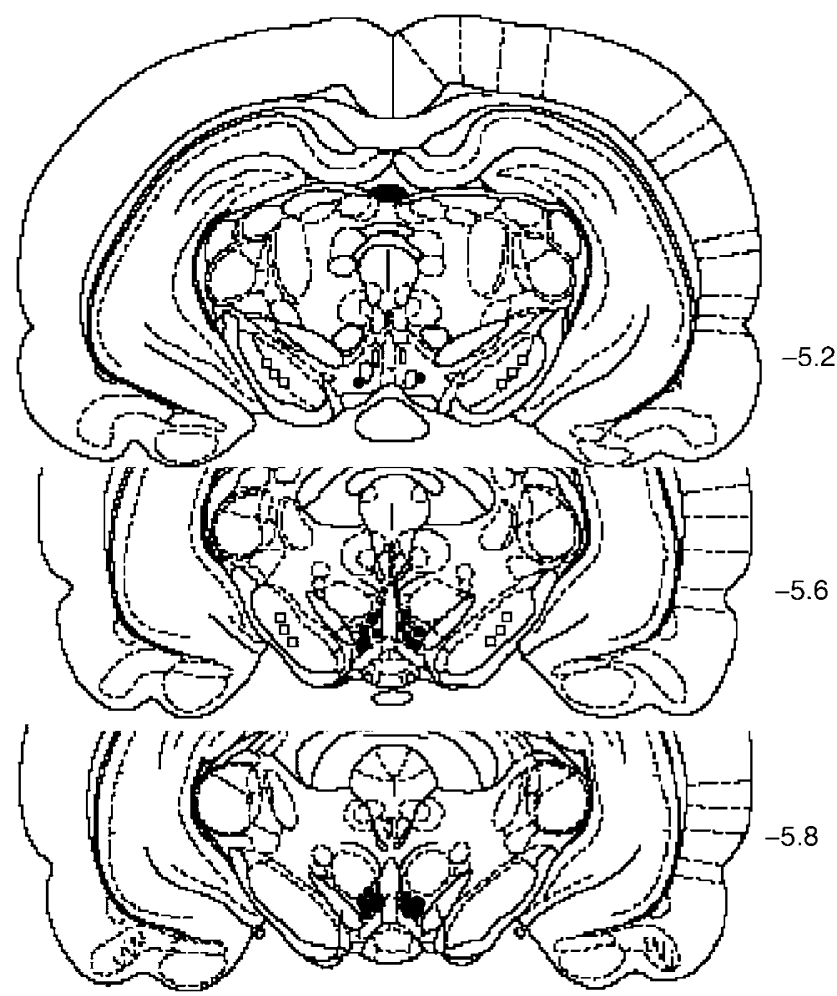

Figure I Schematic depiction of injection sites in the VTA and SN. Coronal brain section images were adapted from the atlas of Paxinos and Watson (1998). Filled circles, squares, and open squares represent the injection sites in sucrose-VTA, cocaine-VTA, and cocaine-SN groups, respectively. five animals. As shown in the lower panel of Figure 2, a repeated, two-way ANOVA revealed that there was a significant time $\times$ dose interaction $\left(\mathrm{F}_{15,72}=2.10\right.$, $P=0.019)$. Bonferroni's tests revealed that at $1 \mu \mathrm{g} / \mathrm{side}$, KYN significantly increased responding compared with saline pretreatment $(P<0.05)$. Although there was a
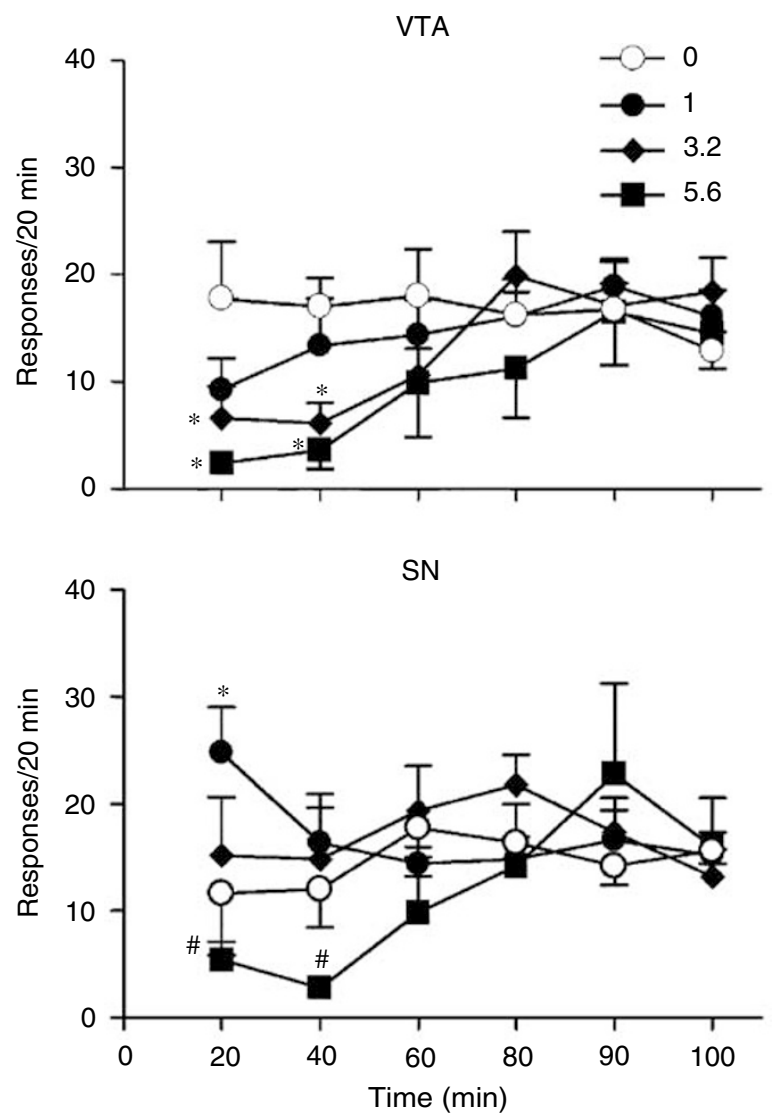

Figure 2 Time-course effects of KYN on cocaine SA maintained under a modified FR 5 schedule. The 2-h session was divided into six, 20-min bins. Data are presented as mean \pm SEM ( $n=8$ for VTA group; $n=5$ for SN group). At $0 \mu \mathrm{g} /$ side $\mathrm{KYN}$, drug vehicle (saline) was given in a volume of $0.5 \mu /$ side. Upper panel: Effects of intra-VTA on cocaine SA. Lower panel: Effects of intra-SN on cocaine SA. There was no significant difference in responding at any time point between the SN and VTA groups after saline pretreatment $\left(F_{5.72}=0.10, P=0.99\right)$. * and \# indicate significantly different $(P<0.01$ and 0.05$)$ from 0 and I $\mu g /$ side, respectively.

Table I Effects of KYN on SA Responding during Timeout Periods

\begin{tabular}{lccc}
\hline & \multicolumn{3}{c}{ Cocaine (responses/2 h) } \\
\cline { 2 - 3 } Dose $(\boldsymbol{\mu g} / \mathbf{s i d e})$ & VTA & SN & \multirow{2}{*}{ Food (responses/min) } \\
\hline 0 & $7 \pm 3.5$ & $3 \pm 1.0$ & $22 \pm 5.4$ \\
1 & $5 \pm 2.5$ & $6 \pm 2.5$ & $9 \pm 2.6 *$ \\
3.2 & $5 \pm 2.2$ & $14 \pm 6.2$ & $5 \pm 1.8^{*}$ \\
5.6 & $3 \pm 1.3$ & $6 \pm 3.8$ & NT \\
\hline
\end{tabular}

Data are group mean \pm SEM. *Significantly different from $0 \mu \mathrm{g} /$ side (repeated one-way ANOVA and Bonferroni's tests, $P<0.05$ and 0.01 , respectively). NT: not tested. 
decreasing trend in responding in the first two bins after $5.6 \mu \mathrm{g} / \mathrm{side}$ compared with saline pretreatment, the effect was not significant. There were, however, significant differences in responding between 1 and $5.6 \mu \mathrm{g} /$ side in the first two bins (Bonferroni's tests, $P<0.05$ ). KYN had no significant effects on responding during timeout periods (Table 1, repeated one-way ANOVA, $\mathrm{F}_{3,15}=1.49, P=0.25$ ).

\section{Experiment 3. Effects of Bilateral Intra-VTA KYN on Cocaine-Primed Reinstatement}

After extinction training, the same group of rats from Experiment 1 was studied for the effects of iGLU receptor blockade on cocaine-primed reinstatement. A repeated, one-way ANOVA revealed that KYN in the VTA dosedependently decreased cocaine-primed reinstatement $\left(\mathrm{F}_{3,21}=18.5, P<0.0001\right)$ compared with saline pretreatment at doses of $3.2 \mu \mathrm{g} / \mathrm{side}$ (Bonferroni's tests, $P<0.001$ ) and $5.6 \mu \mathrm{g} / \mathrm{side}$ (Bonferroni's tests, $P<0.001$ ). There was also a significant difference in responding between 1 and $5.6 \mu \mathrm{g} /$ side (Bonferroni's tests, $P<0.001$ ) or $3.2 \mu \mathrm{g} /$ side (Bonferroni's tests, $P<0.001$ ) (upper panel of Figure 3). Similarly, responding during timeout periods was also decreased at 3.2 and $5.6 \mu \mathrm{g} / \mathrm{side}$ compared with saline pretreatment (Bonferroni's tests, $P<0.01$ ). There was also a significant difference in responding between 1 and $5.6 \mu \mathrm{g} / \mathrm{side}$ (Bonferroni's tests, $P<0.05$ ). Response patterns of a representative rat under the influence of different doses of KYN are shown in Figure 4 (upper panel).

\section{Experiment 4. Effects of Bilateral Intra-SN KYN on Cocaine-Primed Reinstatement}

After extinction training, the same group of rats from Experiment 2 was studied for the effects of iGLU receptor blockade in the SN on cocaine-primed reinstatement. As shown in the lower panel of Figure 3, KYN decreased cocaine-primed reinstatement in the SN group (a repeated, one-way ANOVA, $\mathrm{F}_{3,15}=6.58, P=0.005$ ) compared with saline pretreatment at a dose of $5.6 \mu \mathrm{g} / \mathrm{side}$ (Bonferroni's tests, $P<0.01)$. There was also a significant difference in responding between 1 and $5.6 \mu \mathrm{g} /$ side (Bonferroni's tests, $P<0.05)$. Responding during timeout periods was not significantly decreased at any doses tested, although there was a decreasing trend. Response patterns of a representative rat under the influence of different doses of KYN are shown in Figure 4 (lower panel).

\section{Experiment 5. Effects of Bilateral Intra-VTA KYN on Sucrose SA}

Because $5.6 \mu \mathrm{g} /$ side KYN in the VTA produced an apparent increase in locomotion during cocaine SA and reinstatement, this dose was not tested on sucrose SA or sucroseprimed reinstatement. As shown in the upper panel of Figure 5, KYN decreased sucrose SA significantly (a repeated, one-way ANOVA, $\left.\mathrm{F}_{2,14}=11.3, P=0.001\right)$ compared with saline pretreatment at $3.2 \mu \mathrm{g} /$ side (Bonferroni's tests, $P<0.001)$. Responding during timeout periods was decreased at 1 and $3.2 \mu \mathrm{g} / \mathrm{side}$ compared with saline pretreatment (Table 1, Bonferroni's tests, $P<0.05$ and 0.01 , respectively).
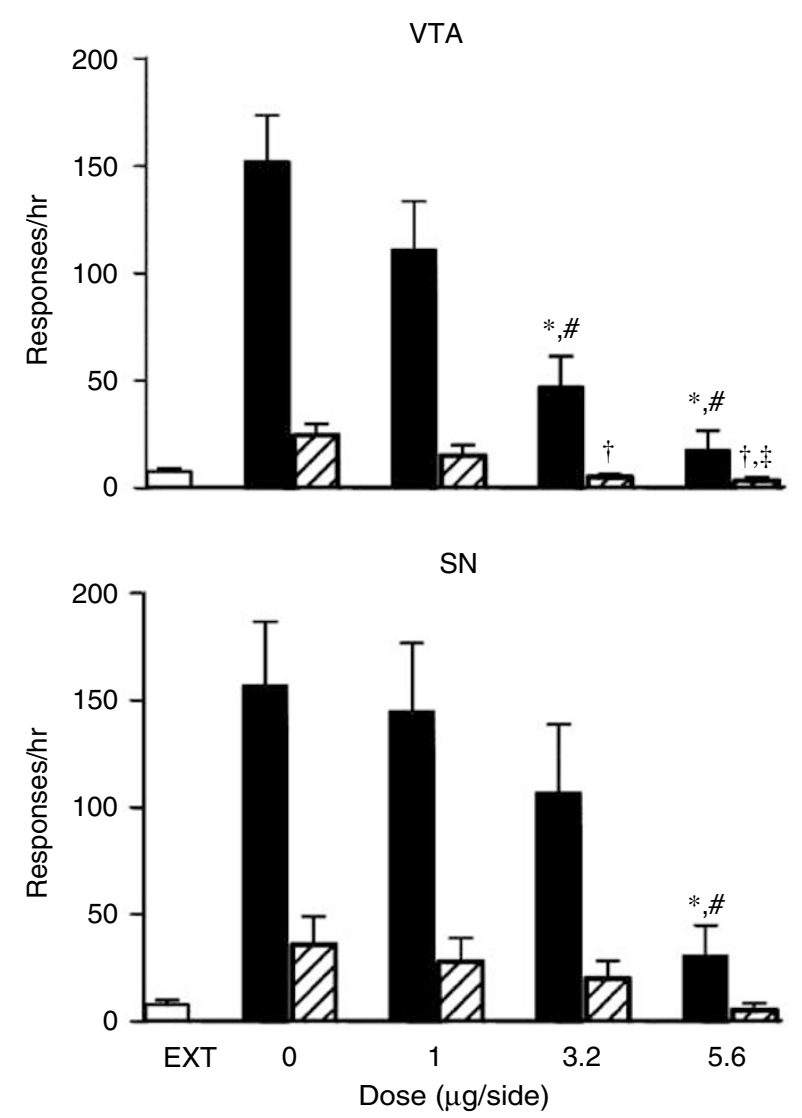

Figure 3 Dose-response effects of KYN on cocaine-primed reinstatement in the presence of cues contingent upon FR 5. Data are presented as mean \pm SEM ( $n=8$ for VTA; $n=6$ for SN). EXT indicates the responses from the extinction training sessions on Thursdays before the test sessions. Solid and hatched bars indicate responses during the session and timeout periods, respectively. Upper panel: Effects of intra-VTA KYN on cocaineprimed reinstatement. Lower panel: Effects of intra-SN KYN on cocaineprimed reinstatement. * and \# indicate significantly different $(P<0.01$ and $0.05)$ from 0 and $I \mu \mathrm{g} /$ side during reinstatement sessions; $\dagger$ and $\ddagger$ indicate significantly different $(P<0.01$ and 0.05$)$ from 0 and $\mid \mu g /$ side during timeout periods, respectively.

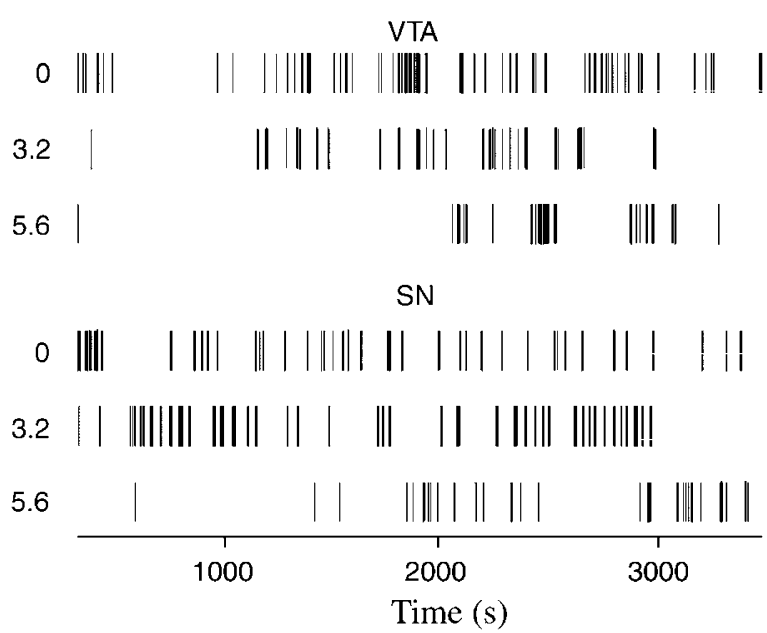

Figure 4 Raster display of the dose-dependent effects of KYN on cocaine-primed reinstatement for representative rats from the VTA and SN groups in the upper and lower panels, respectively. Each short vertical line above the time axis represents a lever press. The numbers on the left side of the raster indicate doses of KYN ( $\mu \mathrm{g} / \mathrm{side})$. 

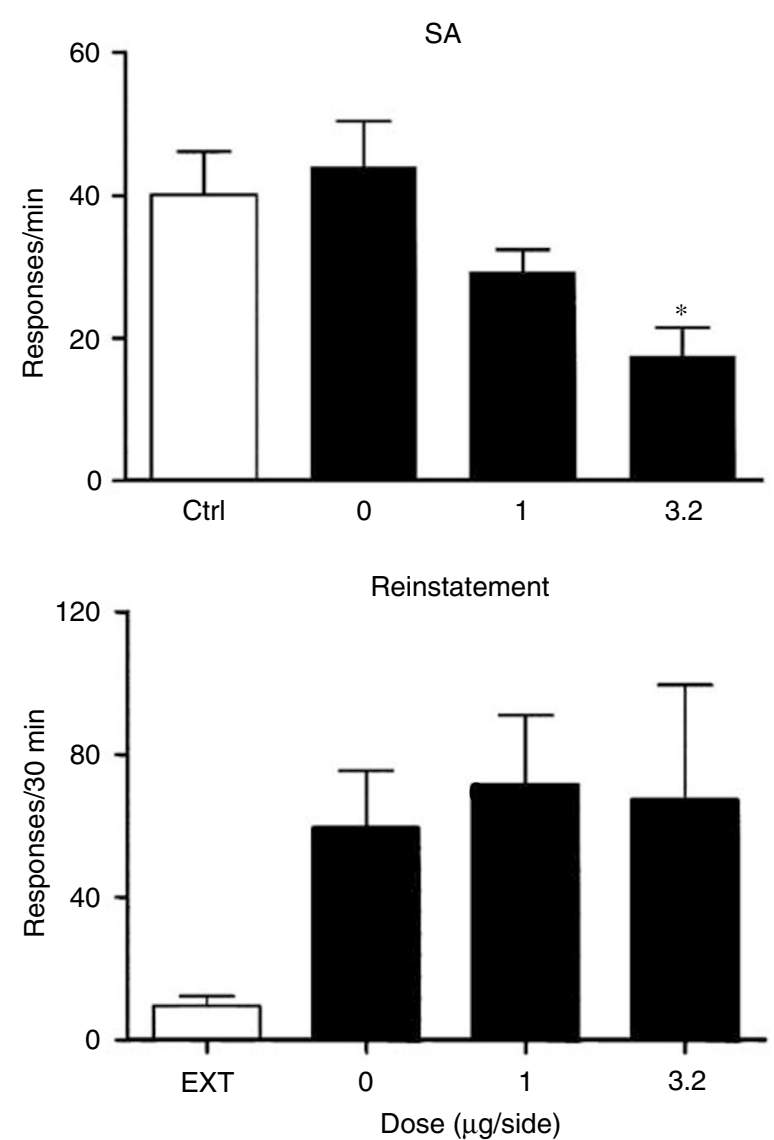

Figure 5 Dose-response effects of intra-VTA KYN on sucrosemaintained or -reinstated responding. Data are presented as mean \pm SEM $(n=8)$. Upper panel: Effects on sucrose SA maintained under FR 5. Ctrl indicates the responses from the sucrose SA training sessions on Thursdays before the test sessions. *Significantly different $(P<0.00 \mathrm{I})$ from $0 \mu \mathrm{g} /$ side. Lower panel: Effects on sucrose-primed reinstatement. EXT indicates the responses from the extinction training sessions on Thursdays before the test sessions. KYN did not significantly decrease responding at any dose tested.

\section{Experiment 6. Effects of Bilateral Intra-VTA KYN on Sucrose-Primed Reinstatement}

After extinction training, the same rats from Experiment 5 were tested for the effects of KYN on sucrose-primed reinstatement. As shown in the lower panel of Figure 5, a repeated, one-way ANOVA revealed that none of the tested doses significantly decreased sucrose-primed reinstatement $\left(\mathrm{F}_{2,14}=0.08, P=0.93\right)$.

\section{DISCUSSION}

Our results indicate that glutamate transmission in the VTA is necessary for cocaine-primed reinstatement since blockade of VTA iGLU receptors with KYN dose-dependently decreased this response. This finding cannot be explained by a nonselective impairment of operant behavior because $3.2 \mu \mathrm{g} /$ side in the VTA failed to affect sucrose-primed operant responding. Diffusion of KYN from the VTA into the $\mathrm{SN}$ is also unlikely because direct infusion of $3.2 \mu \mathrm{g} / \mathrm{side}$ into the $\mathrm{SN}$ failed to affect cocaine-primed reinstatement. In fact, a decrease in cocaine SA in the first two 20-min bins after infusion of this dose into the VTA but not into the SN strongly argues against diffusion as a confounding factor. It appears, therefore, that glutamate in the VTA drives drugprimed relapse to cocaine-seeking behavior.

In contrast, the role of glutamate in the $\mathrm{SN}$ is difficult to interpret. Among the intra-SN doses of KYN tested, only the highest dose $(5.6 \mu \mathrm{g} / \mathrm{side})$ produced a significant decrease in cocaine-primed responding. There are several possible explanations for this effect. First, because DA neurons in the SN are critically important for normal motor function, it is possible that the highest dose of KYN caused a general decline in operant performance. In fact, this dose decreased responding during cocaine $\mathrm{SA}$, although this effect did not reach statistical significance perhaps owing to a small sample size. Because we did not evaluate the effects of intraSN KYN on operant behavior maintained or primed by sucrose pellets, we cannot rule out a motor effect. Second, it is possible that at $5.6 \mu \mathrm{g} / \mathrm{side}$ some $\mathrm{KYN}$ diffuses into the VTA to decrease cocaine-primed responding. A related point is that the injection volume $(0.5 \mu \mathrm{l})$ may be large enough to encroach on the VTA, although an attempt was made to minimize this possibility by separating the SN and the VTA injection sites by at least $0.5 \mathrm{~mm}$. Third, it is possible that the $\mathrm{SN}$ plays a role in cocaine-primed reinstatement. Although temporary inactivation of the SN does not block cocaine-primed reinstatement (McFarland and Kalivas, 2001), the limited amount of data on this topic prevents a definitive conclusion. Our purpose for including the SN infusion group was to determine if the effects of intra-VTA KYN can be explained by diffusion to the SN. Our results at $3.2 \mu \mathrm{g} /$ side rule out this possibility.

Some methodological issues also deserve comment. First, in our cocaine-primed reinstatement test, lever pressing was reinforced by the CS. Because rats trained under the same conditions as the current study showed robust cocaine seeking in response to the CS (Sun and Rebec, 2003), both the CS and cocaine could contribute to the reinstated responding in the present study. The rationale for this protocol is that the CS always plays a role in drug-triggered relapse in human addicts (Childress et al, 1986, 1988). Even if responding were not reinforced by the CS, moreover, we could not completely rule out the possibility that other external cues contribute to reinstatement. It can be argued that although the motivational value of contextual cues is extinguished in the absence of cocaine during extinction training, these cues may regain their motivational value when rats are tested under the influence of cocaine (statedependent effects). Thus, unless the reinstatement test is conducted in a different environment in the absence of discrete cues, it is unlikely that the effects of external cues on cocaine-primed reinstatement can be completely eliminated. We did not attempt to distinguish among these reinstatement triggers because in human addicts druginduced craving or relapse is always accompanied by the CS (Childress et al, 1986, 1988) and the CS as conditioned reinforcers may be very important in sustaining drugseeking behavior. Increased DA in the NAcc has been implicated in cue-induced reinstatement (Taylor and Robbins, 1984; Robinson and Berridge, 2003). Given the important role of DA in the DPFC and NAcc in cocaineseeking behavior induced by cocaine and cocaine-paired 
cues, respectively, blockade of iGLU in the VTA should decrease DA input to both regions and thereby decrease both processes. It is unlikely that the differential effects of KYN on cocaine- and food-seeking behaviors are only due to blockade of the cue effects in cocaine-primed reinstatement. A second methodological issue is that higher levels of cocaine-primed responding were observed in the present experiment compared to those in the literature (McFarland and Kalivas, 2001;Park et al, 2002;Fuchs et al, 2004). The difference may be related to training doses, reinstatement schedules, training histories, and whether responding is reinforced by the CS. Finally, repeated microinjections may damage the injection site and thus confound the results. Inspection of brain slices, however, did not reveal extensive gliosis surrounding the injection sites. It is unclear, moreover, to what degree mechanical damage may affect cocaine-seeking and cocaine-taking behavior. Since the order of doses including vehicle was counterbalanced, a potential lesion cannot explain our dose-dependent effects of KYN.

Blockade of NMDA receptors in the VTA increases locomotion in rats (Cornish et al, 2001), a finding consistent with our informal observation that intra-VTA KYN at $5.6 \mu \mathrm{g}$ / side increased locomotion. Thus, one could argue that this change in locomotion may have confounded our results by interfering with operant behavior. This explanation, however, is at odds with our finding that at $3.2 \mu \mathrm{g} /$ side intra-VTA KYN decreased cocaine-primed reinstatement but failed to affect sucrose-seeking responding induced by sucrose. In fact, this finding supports evidence that the VTA is not critically involved in food-primed food-seeking behavior (McFarland and Kalivas, 2001). Another issue to consider is the stereotyped pattern of motor activation induced by cocaine (Ushijima et al, 1995). It is conceivable, for example, that during cocaine SA, lever pressing becomes part of a stereotyped motor response. We found, however, that stereotyped activity induced by cocaine occurred away from the lever and was manifest as burst grooming and head bobbing. No persistent bar pressing was observed, although a form of this behavior was seen in a previous study (Sun and Rebec, 2005). It appears, therefore, that KYN in the VTA acted directly on the mechanisms underlying drug-primed reinstatement and not on the neural substrates of movement.

The source of the glutamate input after cocaine priming remains to be determined. Two potential brain stem sources, the midbrain tegmental nuclei and the bed nucleus of the stria terminalis (BNST), have not been studied for a role in drug-primed reinstatement. Although the amygdala, which sends glutamate fibers directly to the VTA, is crucial for cue-induced reinstatement (Meil and See, 1997; Everitt et al, 2001; Fuchs et al, 2002), amygdaloid involvement in cocaine priming is controversial (McFarland and Kalivas, 2001; Kantak et al, 2002). The ventral subiculum (vSUB), moreover, is an unlikely source since no direct connections to the VTA have been identified. It is interesting, however, that the vSUB projects directly to the PFC (Jay and Witter, 1991), which in turn projects to the VTA (Sesack et al, 2003). Thus, vSUB stimulation could act via the PFC to increase VTA glutamate. Because both the PFC and VTA have been implicated in cocaine-primed reinstatement, it is important to know how these two structures interact to elicit this response.
There are reciprocal connections between the PFC and VTA (Sesack et al, 2003). DA neurons innervated by PFC project back to PFC (Carr and Sesack, 2000). This specific connectivity provides a direct feedback mechanism between the PFC and VTA. Because cocaine-primed reinstatement depends on increased glutamate inputs from the PFC to the NAcc (Baker et al, 2003; McFarland et al, 2003) as well as DA input to PFC (McFarland and Kalivas, 2001; Park et al, 2002), PFC DA may be critical for the glutamate response. It is tempting to suggest, therefore, that an increase in PFC DA after cocaine priming may enhance the activity of PFC output neurons and thereby drive drug-seeking behavior. Although there is no convincing evidence that DA strongly excites PFC output neurons, it appears to modulate such activity. For example, the resting membrane potentials of PFC output neurons alternate between an 'up' state when they can generate impulses and a 'down' state when they are silent (Lewis and O'Donnell, 2000; Peters et al, 2000). DA appears to switch neurons from the 'down' to the 'up' state and to increase resident time in the 'up' state (Lewis and O'Donnell, 2000; Lavin and Grace, 2001). In fact, the 'up' state is synchronized with VTA activity (Peters et al, 2004). Consequently, DA can increase the chance for output neurons to fire and prolong the duration of such activity at a given excitatory input in the PFC. Another interesting observation is that PFC regulates the activity of VTA neurons (Takahata and Moghaddam, 1998; AlmodovarFabregas et al, 2002). It is conceivable, therefore, that increased DA after cocaine priming may increase PFC output activity, which, in turn, activates VTA DA neurons and thus helps to sustain activity in PFC outputs. This positive interaction between PFC and VTA may underlie persistent drug-seeking behavior after cocaine priming.

The VTA also sends DA terminals to the NAcc where it is involved in cocaine reinforcing effects (Maldonado et al, 1993; McGregor and Roberts, 1993; Phillips et al, 2003). It is conceivable that blockade of VTA iGLU receptors decreases cocaine-primed reinstatement by interfering with DA in the NAcc. The evidence for the role of NAcc DA in cocaineprimed reinstatement is inconsistent. Although direct blockade of DA receptors in NAcc core fails to decrease cocaine-primed reinstatement, microinjection of DA into the core reinstates cocaine-seeking behavior (McFarland and Kalivas, 2001; Anderson et al, 2003; Cornish and Kalivas, 2000). Because cocaine itself increases extracellular DA, which is sufficient to induce cocaine-seeking behavior, it is unclear how to reconcile these results. Vezina (2004) proposes that reinstatement of drug-seeking behavior is not dependent on NAcc DA, the expression of enhanced reinstatement is. The evidence for the involvement of the NAcc shell is also inconsistent. For example, inactivation of the shell does not affect cocaine-primed reinstatement, whereas blockade of shell D1 DA receptors does (McFarland and Kalivas, 2001; Anderson et al, 2003). Further investigations of this issue are warranted.

Although the specific involvement of VTA iGLU receptors in cocaine SA has yet to be studied, there is evidence implicating the VTA in such behavior (Ranaldi and Wise, 2001; Ikemoto and Wise, 2002). It is possible, therefore, that blockade of iGLU receptors in the VTA decreases cocaine-primed reinstatement by interfering with cocaine reinforcing effects. Indeed, at the dose that decreased 
cocaine-primed reinstatement, KYN also decreased cocaine SA. Intra-VTA KYN blocks the activation of VTA DA neurons induced by electrical stimulation of the BNST or chemical stimulation of the vSUB (Legault et al, 2000; Georges and Aston-Jones, 2002). In addition, the same studies demonstrate that the increase in DA efflux in the NAcc induced by chemical stimulation of the vSUB is blocked by intra-VTA KYN and that intra-VTA KYN alone does not increase either VTA DA neuronal activity or DA efflux in the NAcc. Together, these data suggest that KYN decreases the reinforcing effects of cocaine by blocking the increase in NAcc DA transmission during cocaine SA. Thus, one explanation for the effect of KYN on cocaine-primed reinstatement observed here is that KYN decreases the reinforcing effects of cocaine. Interestingly, however, some iGluR antagonists alone produce reinforcing effects in the VTA (Harris and Aston-Jones, 2003; David et al, 1998). Although this finding leaves open the possibility that KYN may potentiate cocaine reinforcement and thereby decrease cocaine SA by a satiety mechanism, we have already pointed out that intra-VTA KYN does not enhance DA transmission (see above). Thus, if there is a satiety effect, it must operate independently of DA. Noteworthy in this regard is evidence that intra-VTA NMDA receptor antagonists increase locomotion by reducing the tonic activity of GABAergic neurons that project to the forebrain resulting in forebrain disinhibition (Cornish et al, 2001). The same mechanism may underlie the reinforcing effects of iGluR antagonists in the VTA. Satiation caused by enhanced cocaine reinforcement, moreover, is unlikely to explain the effects of intra-VTA KYN on reinstatement given that intra-VTA KYN blocks cocaineseeking behavior induced by electrical stimulation of the vSUB and that microinjections of NMDA, not KYN, into the VTA reinstate cocaine-seeking behavior (Vorel et al, 2001).

Recently, it has been suggested that blockade of iGluR in the VTA blocks the ability of rats to associate cocaine with the environment (Harris and Aston-Jones, 2003). Thus, the cueing effects of cocaine-conditioned contextual stimuli may require activation of the VTA via iGluR. One could argue, therefore, that the effect of intra-VTA KYN on reinstatement is due to blockade of the cueing effects of the CS. Note, however, that robust cocaine-seeking behavior can be reinstated by cocaine alone in the absence of the conditioned cues (McFarland et al, 2003; McFarland and Kalivas, 2001). Thus, our observation of a robust decrease in cocaine-seeking behavior after cocaine priming cannot be explained solely by a blockade of cueing effects.

In summary, our results demonstrate that blockade of VTA iGLU receptors specifically decreased cocaine-primed reinstatement, suggesting that increased glutamate inputs into the VTA after cocaine priming is critical for reinstatement. Further investigations into the role of brain regions that innervate the VTA will enhance our understanding of the neural substrates of cocaine relapse.

\section{ACKNOWLEDGEMENTS}

This research was supported by NIH (DA02451). We thank Paul Langley and Faye Caylor for technical and administrative support. There is no conflict of interest in connection with the manuscript with any association or organization.

\section{REFERENCES}

Almodovar-Fabregas LJ, Segarra O, Colon N, Dones JG, Mercado M, Mejias-Aponte CA et al (2002). Effects of cocaine administration on VTA cell activity in response to prefrontal cortex stimulation. Ann NY Aca Sci 965: 157-171.

Anderson SM, Bari AA, Pierce RC (2003). Administration of the D1-like dopamine receptor antagonist $\mathrm{SCH}-23390$ into the medial nucleus accumbens shell attenuates cocaine priminginduced reinstatement of drug-seeking behavior in rats. Psychopharmacology 168: 132-138.

Baker DA, McFarland K, Lake RW, Shen H, Tang XC, Toda S et al (2003). Neuroadaptations in cystine-glutamate exchange underlie cocaine relapse. Nat Neurosci 6: 743-749.

Breiter HC, Gollub RL, Weisskoff RM, Kennedy DN, Makris N, Berke JD et al (1997). Acute effects of cocaine on human brain activity and emotion. Neuron 19: 591-611.

Caine SB, Negus SS, Mello NK, Bergman J (1999). Effects of dopamine $\mathrm{D}$ (1-like) and $\mathrm{D}$ (2-like) agonists in rats that self-administer cocaine. J Pharmacol Exp Ther 291: 353-360.

Capriles N, Rodaros D, Sorge RE, Stewart J (2003). A role for the prefrontal cortex in stress- and cocaine-induced reinstatement of cocaine seeking in rats. Psychopharmacology 168: $66-74$.

Carr DB, Sesack SR (2000). Projections from the rat prefrontal cortex to the ventral tegmental area: target specificity in the synaptic associations with mesoaccumbens and mesocortical neurons. J Neurosci 20: 3864-3873.

Childress AR, McLellan AT, Ehrman R, O’Brien CP (1988). Classically conditioned responses in opioid and cocaine dependence: a role in relapse? NIDA Res Monogr 84: 25-43.

Childress AR, McLellan AT, O'Brien CP (1986). Role of conditioning factors in the development of drug dependence. Psychiatr Clin N Am 9: 413-425.

Childress AR, Mozley PD, McElgin W, Fitzgerald J, Reivich M, O’Brien CP (1999). Limbic activation during cue-induced cocaine craving. Am J Psychiatry 156: 11-18.

Cornish JL, Kalivas PW (2000). Glutamate transmission in the nucleus accumbens mediates relapse in cocaine addiction. $J$ Neurosci 20: RC89.

Cornish JL, Nakamura M, Kalivas PW (2001). Dopamineindependent locomotion following blockade of $\mathrm{N}$-methyl-Daspartate receptors in the ventral tegmental area. J Pharmacol Exp Ther 298: 226-233.

David V, Durkin TP, Cazala P (1998). Rewarding effects elicited by the microinjection of either AMPA or NMDA glutamatergic antagonists into the ventral tegmental area revealed by an intracranial self-administration paradigm in mice. Eur J Neurosci 10: $1394-1402$.

Everitt BJ, Dickinson A, Robbins TW (2001). The neuropsychological basis of addictive behaviour. Brain Res Brain Res Rev 36: 129-138.

Fuchs RA, Evans KA, Parker MP, See RE (2004). Differential involvement of orbitofrontal cortex subregions in conditioned cue-induced and cocaine-primed reinstatement of cocaine seeking in rats. $J$ Neurosci 24: 6600-6610.

Fuchs RA, Weber SM, Rice HJ, Neisewander JL (2002). Effects of excitotoxic lesions of the basolateral amygdala on cocaineseeking behavior and cocaine conditioned place preference in rats. Brain Res 929: 15-25.

Georges F, Aston-Jones G (2002). Activation of ventral tegmental area cells by the bed nucleus of the stria terminalis: a novel excitatory amino acid input to midbrain dopamine neurons. J Neurosci 22: 5173-5187.

Grant S, London ED, Newlin DB, Villemagne VL, Liu X, Contoreggi $C$ et al (1996). Activation of memory circuits during cue-elicited cocaine craving. Proc Natl Acad Sci 93: 12040-12045. 
Harris GC, Aston-Jones G (2003). Critical role for ventral tegmental glutamate in preference for a cocaine-conditioned environment. Neuropsychopharmacology 28: 73-76.

Hyman SE, Malenka RC (2001). Addiction and the brain: the neurobiology of compulsion and its persistence. Nat Rev Neurosci 2: 695-703.

Ikemoto S, Wise RA (2002). Rewarding effects of the cholinergic agents carbachol and neostigmine in the posterior ventral tegmental area. J Neurosci 22: 9895-9904.

Jay TM, Witter MP (1991). Distribution of hippocampal CA1 and subicular efferents in the prefrontal cortex of the rat studied by means of anterograde transport of phaseolus vulgaris-leucoagglutinin. J Comp Neurol 313: 574-586.

Johnson SW, North RA (1992). Opioids excite dopamine neurons by hyperpolarization of local interneurons. J Neurosci 12: 483-488.

Kantak KM, Black Y, Valencia E, Green-Jordan K, Eichenbaum HB (2002). Dissociable effects of lidocaine inactivation of the rostral and caudal basolateral amygdala on the maintenance and reinstatement of cocaine-seeking behavior in rats. J Neurosci 22: $1126-1136$.

Kilts CD, Schweitzer JB, Quinn CK, Gross RE, Faber TL, Muhammad F et al (2001). Neural activity related to drug craving in cocaine addiction. Arch Gen Psychiatry 58: 334-341.

Lavin A, Grace AA (2001). Stimulation of D1-type dopamine receptors enhances excitability in prefrontal cortical pyramidal neurons in a state-dependent manner. Neuroscience 104: 335-346.

Legault M, Rompre Pierre-Paul, Wise RA (2000). Chemical stimulation of the hippocampus elevates nucleus accumbens dopamine by activating dopaminergic neurons of the ventral tegmental area. J Neurosci 20: 1635-1642.

Leshner AI, Koob GF (1999). Drugs of abuse and the brain. Proc Assoc Am Physicians 111: 99-108.

Lewis BL, O'Donnell P (2000). Ventral tegmental area afferents to the prefrontal cortex maintain membrane potential 'up' states in pyramidal neurons via $\mathrm{D}(1)$ dopamine receptors. Cereb Cortex 10: $1168-1175$.

Maas LC, Lukas SE, Kaufman MJ, Weiss RD, Daniels SL, Rogers VW et al (1998). Functional magnetic resonance imaging of human brain activation during cue-induced cocaine craving. $\mathrm{Am}$ J Psychiatry 155: 124-126.

Maldonado R, Robledo P, Chover AJ, Caine SB, Koob GF (1993). D1 dopamine receptors in the nucleus accumbens modulate cocaine self-administration in the rat. Pharmacol Biochem Behav 45: 239-242.

McClung CA, Nestler EJ (2003). Regulation of gene expression and cocaine reward by CREB and DeltaFosB. Nat Neurosci 6: $1208-1215$.

McFarland K, Kalivas PW (2001). The circuitry mediating cocaineinduced reinstatement of drug-seeking behavior. J Neurosci 21: 8655-8663.

McFarland K, Lapish CC, Kalivas PW (2003). Prefrontal glutamate release into the core of the nucleus accumbens mediates cocaineinduced reinstatement of drug-seeking behavior. J Neurosci 23: 3531-3537.

McGregor A, Roberts DC (1993). Dopaminergic antagonism within the nucleus accumbens or the amygdala produces differential effects on intravenous cocaine self-administration under fixed and progressive ratio schedules of reinforcement. Brain Res 624: 245-252.

McLaughlin J, See RE (2003). Selective inactivation of the dorsomedial prefrontal cortex and the basolateral amygdala attenuates conditioned-cued reinstatement of extinguished cocaine-seeking behavior in rats. Psychopharmacology 168: $57-65$.
Meil WM, See RE (1997). Lesions of the basolateral amygdala abolish the ability of drug associated cues to reinstate responding during withdrawal from self-administered cocaine. Behav Brain Res 87: 139-148.

Nestler EJ (2004). Historical review: molecular and cellular mechanisms of opiate and cocaine addiction. Trends Pharmacol Sci 25: 210-218.

Park WK, Bari AA, Jey AR, Anderson SM, Spealman RD, Rowlett JK et al (2002). Cocaine administered into the medial prefrontal cortex reinstates cocaine-seeking behavior by increasing AMPA receptor-mediated glutamate transmission in the nucleus accumbens. J Neurosci 22: 2916-2925.

Paxinos G, Watson C (1998). The Rat Brain in Stereotaxic Coordinates, 4th edn. Academic Press: San Diego.

Peters Y, Barnhardt NE, O’Donnell P (2004). Prefrontal cortical up states are synchronized with ventral tegmental area activity. Synapse 52: 143-152.

Peters YM, Lewis BL, O’Donnell P (2000). Synchronous activity in the ventral tegmental area and prefrontal cortex. Ann NY Aca Sci 909: 267-269.

Phillips PE, Stuber GD, Heien ML, Wightman RM, Carelli RM (2003). Subsecond dopamine release promotes cocaine seeking. [see comment] [erratum appears in Nature 2003 May 22;423(6938):461]. Nature 422: 614-618.

Ranaldi R, Wise RA (2001). Blockade of D1 dopamine receptors in the ventral tegmental area decreases cocaine reward: possible role for dendritically released dopamine. J Neurosci 21: 58415846.

Robinson TE, Berridge KC (2003). Addiction. Annu Rev Psychol 54: 25-53.

Sesack SR, Carr DB, Omelchenko N, Pinto A (2003). Anatomical substrates for glutamate-dopamine interactions: evidence for specificity of connections and extrasynaptic actions. Ann NY Aca Sci 1003: 36-52.

Stewart J (1984). Reinstatement of heroin and cocaine selfadministration behavior in the rat by intracerebral application of morphine in the ventral tegmental area. Pharmacol Biochem Behav 20: 917-923.

Stone TW (1993). Neuropharmacology of quinolinic and kynurenic acids. Neuropharmacology 45: 309-379.

Sun W, Rebec GV (2003). Lidocaine inactivation of ventral subiculum attenuates cocaine-seeking behavior in rats. $J$ Neurosci 23: 10258-10264.

Sun W, Rebec GV (2005). The role of prefrontal cortex D1-like and D2-like receptors in cocaine-seeking behavior in rats. Psychopharmacology 177: 315-323.

Takahata R, Moghaddam B (1998). Glutamatergic regulation of basal and stimulus-activated dopamine release in the prefrontal cortex. J Neurochem 71: 1443-1449.

Taylor JR, Robbins TW (1984). Enhanced behavioural control by conditioned reinforcers following microinjections of D-amphetamine into the nucleus accumbens. Psychopharmacology 84: 405-412.

Ungless MA, Whistler JL, Malenka RC, Bonci A (2001). Single cocaine exposure in vivo induces long-term potentiation in dopamine neurons. Nature 411: 583-587.

Ushijima I, Carino MA, Horita A (1995). Involvement of D1 and D2 dopamine systems in the behavioral effects of cocaine in rats. Pharmacol Biochem Behav 52: 737-741.

Vezina P (2004). Sensitization of midbrain dopamine neuron reactivity and the self-administration of psychomotor stimulant drugs. Neurosci Biobehav Rev 27: 827-839.

Vorel SR, Liu X, Hayes RJ, Spector JA, Gardner EL (2001). Relapse to cocaine-seeking after hippocampal theta burst stimulation. Science 292: 1175-1178. 